\title{
The millimeter-wave properties of superconducting microstrip lines
}

\author{
A. Vayonakis* , C. Luo $^{\dagger}$, H.G. Leduc** ${ }^{* *}$ R. Schoelkopf ${ }^{\ddagger}$ and J. Zmuidzinas* \\ ${ }^{*}$ California Institute of Technology, Pasadena, $C A$ \\ ${ }^{\dagger}$ Massachusetts Institute of Technology, Cambridge, MA \\ ${ }^{* *}$ Jet Propulsion Laboratory, Pasadena, $C A$ \\ ${ }^{\ddagger}$ Yale University, New Haven, CT
}

\begin{abstract}
We have developed a novel technique for making high quality measurements of the millimeter-wave properties of superconducting thin-film microstrip transmission lines. Our experimental technique currently covers the $75-100 \mathrm{GHz}$ band. The method is based on standing wave resonances in an open ended transmission line. We obtain information on the phase velocity and loss of the microstrip. Our data for $\mathrm{Nb} / \mathrm{SiO} / \mathrm{Nb}$ lines, taken at $4.2 \mathrm{~K}$ and $1.6 \mathrm{~K}$, can be explained by a single set of physical parameters. Our preliminary conclusion is that the loss is dominated by the $\mathrm{SiO}$ dielectric, with a temperature-independent loss tangent of $5.3 \pm 0.5 \times 10^{-3}$ for our samples.
\end{abstract}

\section{INTRODUCTION}

Superconducting microstrip lines are of major importance for tuning elements in SIS mixers [1]. Microstrip lines have also been proposed for use in millimeter and submillimeter direct detection applications, such as antenna coupled bolometer arrays with on-chip band-pass filters. Such highly integrated architectures would allow for a very large number of pixels and would enable novel instruments, such as a multiband imaging polarimeter or an on-chip spectrometer. The transmission losses of superconducting microstrip lines are a key issue for the feasibility of such architectures. The properties of superconducting microstrip lines have been investigated previously [2, 3]. However, the method that we present here provides much higher quality data.

\section{EXPERIMENTAL METHOD}

Our method is based on standing wave resonances in an open ended microstrip stub. The entire circuit is fabricated on a thick silicon substrate $(400 \mu \mathrm{m})$, and the millimeter-wave radiation is coupled onto the chip quasi-optically using a silicon substrate lens [1]. There are two $\mathrm{Nb} / \mathrm{Al}$-oxide/ $\mathrm{Nb}$ SIS junctions on the chip which serve as direct detectors. One of the junctions is connected to an open-ended $\mathrm{Nb} / \mathrm{SiO} / \mathrm{Nb}$ microstrip stub (Figure 1). Having two SIS junctions on the chip allows us to calibrate out changes in the power that is coupled onto the chip from the external signal source. The ratio of the signal from the junction connected to the stub, to the signal from the "power reference" junction, gives us a precise relative power response, whose frequency dependence carries information

CP605, Low Temperature Detectors, edited by F. S. Porter et al. (C) 2002 American Institute of Physics 0-7354-0049-0/02/\$19.00 539 


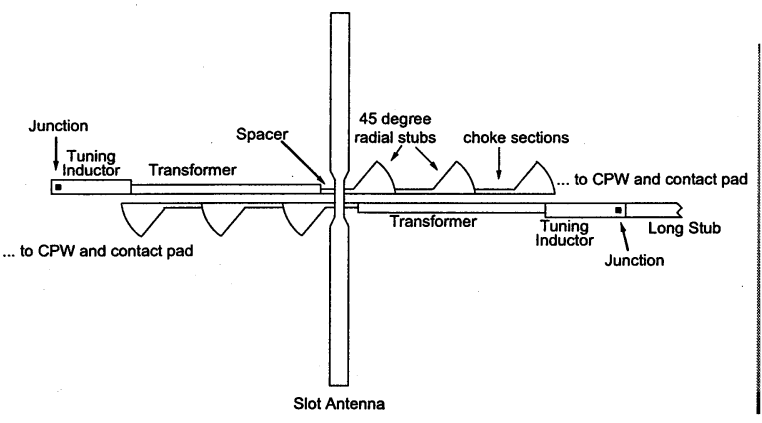

(a)

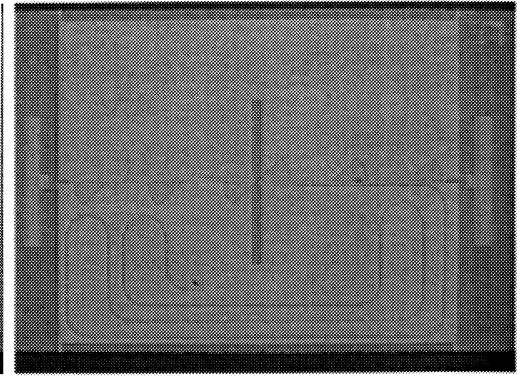

(b)

FIGURE 1. (a) Schematic diagram of chip. (b) Photograph of a chip with a $1 \mathrm{~cm}$ long microstrip stub. The top strip of the microstrip is $5 \mu \mathrm{m}$ wide, and the thicknesses of the $\mathrm{Nb}$ top strip, SiO dielectric, and $\mathrm{Nb}$ ground plane layers are $2000 \AA$ each.

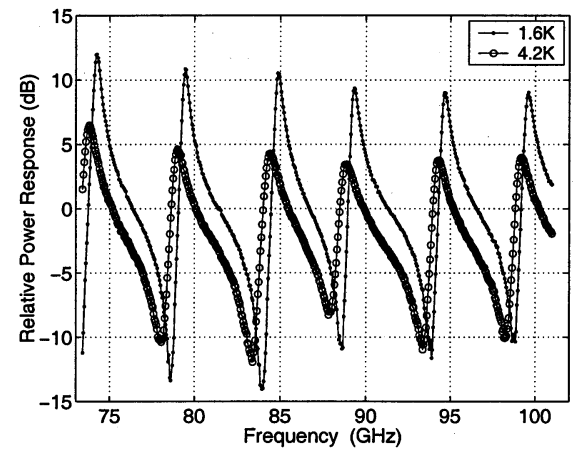

(a)

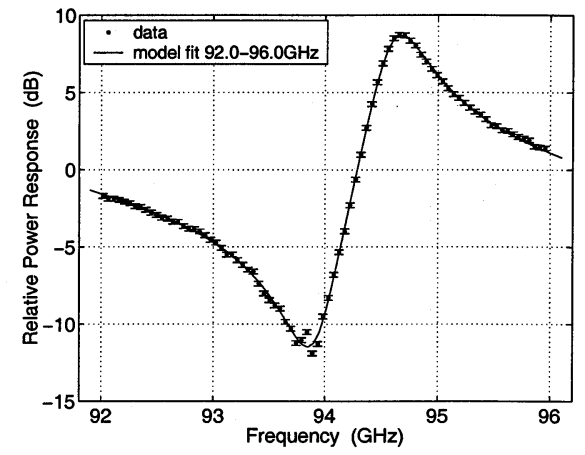

(b)

FIGURE 2. (a) Microstrip power response vs. frequency at two different temperatures and $2.00 \mathrm{mV}$ bias. The loss decreases and the phase velocity increases at the lower temperature, in agreement with theoretical expectations. (b) Example fit of $2.00 \mathrm{mV}$ bias data, at $4.2 \mathrm{~K}$.

about the properties of the microstrip stub.

We use two harmonic generators as radiation sources. These harmonic generators are driven by two microwave signal generators $(10-18 \mathrm{GHz})$, and produce a series of coherent millimeter wave frequencies that are exact integer multiples of the input signal frequencies. The two microwave signals differ in frequency by a small value (typically $10 \mathrm{MHz}$ ), and this difference is multiplied with harmonic number. The two SIS junctions mix these signals and convert the millimeter waves to signals at various RF beat frequencies. By detecting the output signals from the junctions, that are locked to a specific beat frequency, we select out a unique harmonic number and therefore measure the microstrip response at the corresponding frequency. Figure 2(a) shows representative data taken at two different temperatures. 


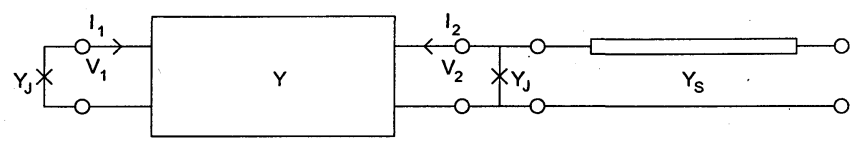

FIGURE 3. Theoretical model schematic diagram.

\section{Theoretical Model}

We treat the symmetric part of the circuit as a two-port black box linear circuit with unknown admittance matrix $Y$, which includes the antenna, the junction capacitance, and the matching circuits (Figure 3). The admittance matrix $Y$ does not include the admittance of the junction due to the tunneling currents, $Y_{J}$, or the microstrip stub. The relative power response is given by the detected power ratio of the two junctions which can be shown to be:

$$
P_{d B}(v ; K, \tau, w)=10 \log _{10}\left|\frac{V_{2}}{V_{1}}\right|^{2}=-20 \log _{10}\left|1+w \frac{1-\tau \exp (-2 i K v)}{1+\tau \exp (-2 i K v)}\right| .
$$

Here $L$ is the length of the stub, $K=\frac{2 \pi L}{\bar{c}}$ is the phase constant, $\tau=\exp (-2 \alpha L)$ is the round trip amplitude attenuation, and $w$ is a complex coefficient which depends on the admittances of the circuit elements. We assume that these quantities are essentially constant over the narrow $(4 \mathrm{GHz})$ frequency range of a single resonance, and obtain their values using a least-squares fit to the data. Figure 2(b) shows an example fit of (1) over a $4 \mathrm{GHz}$ frequency range.

\section{RESULTS}

We have taken data in the $75-100 \mathrm{GHz}$ band, at $4.2 \mathrm{~K}$ and $1.6 \mathrm{~K}$, for a wide range of junction bias voltages both below and above the gap voltage. Figure 4(a) shows the measured loss at the two temperatures (for four frequency bins). Although the junction impedance varies greatly from $2.0 \mathrm{mV}$ to $3.6 \mathrm{mV}$ (Figures 5(b),(c)), the extracted values of the loss are essentially independent of bias voltage. This demonstrates that, given the quality of our data, our data analysis technique can reliably separate out the effects of microstrip loss. The difference of about $2 \%$ power loss per wavelength between the two temperatures is accounted for by the theoretical (Mattis-Bardeen) loss difference in the superconductor. The remaining loss is attributed to the dielectric, with a temperatureindependent loss tangent $\tan \delta_{S i O}=5.3 \pm 0.5 \times 10^{-3}$ for our samples. Figure 4(b) shows the measured phase velocity at the two temperatures.

We have also observed a hysteretic behavior of the loss as a function of the magnetic field strength. In Figure 5(a), the loss vs. magnetic field current (which is proportional to the magnetic field) is shown. We attribute this loss and the "memory" effect (hysteresis) to the creation of fluxons in the superconducting films.

Figures 5(b),(c) show the quantum conductance (susceptance) of the junctions vs. bias voltage $[4,5]$. The solid lines are calculated from the DC I-V curve. The data points are 


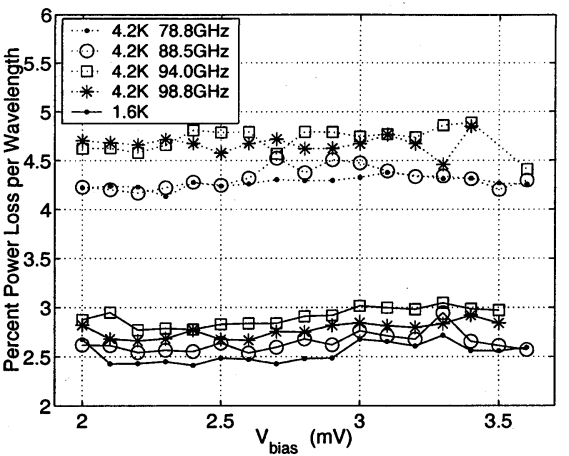

(a)

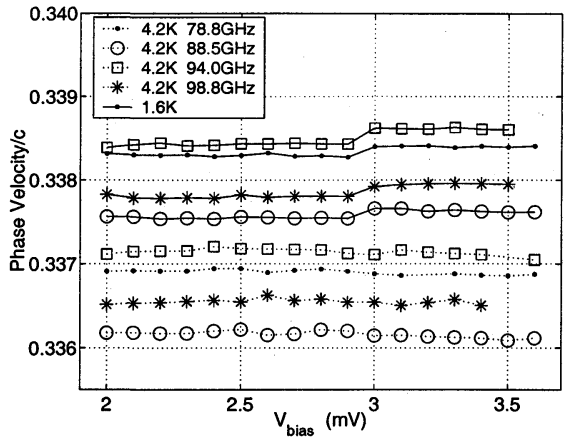

(b)

FIGURE 4. Experimental results at $4.2 \mathrm{~K}$ and $1.6 \mathrm{~K}$, for four frequency values. (a) Percent power loss per wavelength vs. junction bias voltage. (b) Phase velocity vs. junction bias voltage.

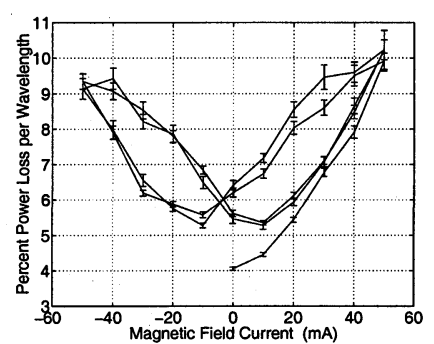

(a)

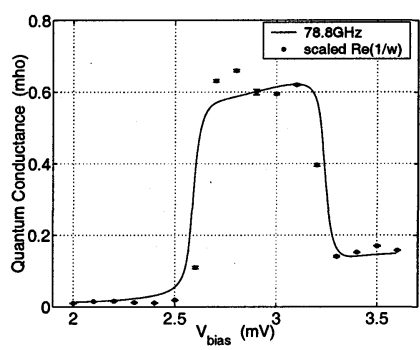

(b)

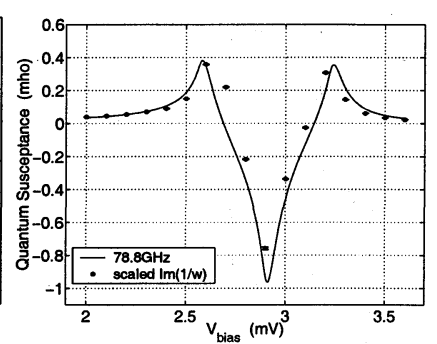

(c)

FIGURE 5. (a) Power loss per wavelength vs. magnetic field current. The junctions are biased at 2.50 $\mathrm{mV}$, and the first point is taken with the magnetic field turned off. Subsequent points were taken with magnetic field current stepped by $10 \mathrm{~mA}$, ranging between $-50 \mathrm{~mA}$, and $+50 \mathrm{~mA}$. (b,c) see text.

the real (imaginary) parts of $1 / w$, which scales as the admittance of the junction $Y_{J}$.

\section{REFERENCES}

1. Gaidis, M., LeDuc, H., Bin, M., Miller, D., Stern, J., and Zmuidzinas, J., IEEE transactions on Microwave Theory and Techniques, 44, 1130-1139 (1996).

2. Bi, B., Wan, K., Zhang, W., Han, S., and Lukens, J., IEEE Transactions on Applied Superconductivity, 1, 145-149 (1991).

3. Currie, M., Sobolewski, R., and Hsiang, T., Applied Physics Letters, 73, 1910-1912 (1998).

4. Hu, Q., Mears, C., Richards, P., and Lloyd, F., Physical Review Letters, 64, 2945-2948 (1990).

5. Worsham, A., Ugras, N., Winkler, D., and Prober, D., Physical Review Letters, 67, 3034-3037 (1991). 